ISSN 0103-5150

Fisioter. Mov., Curitiba, v. 24, n. 3, p. 379-388, jul./set. 2011 Licenciado sob uma Licença Creative Commons

\title{
Exercício aeróbio e fortalecimento muscular melhoram o desempenho funcional na doença de Parkinson
}

\author{
Aerobic exercise and muscular strengthening improve functional \\ performance in Parkinson's disease
}

\author{
Fátima Rodrigues-de-Paula ${ }^{[a]}$, Lidiane Oliveira Lima ${ }^{[b]}$, Luci Fuscaldi Teixeira-Salmela ${ }^{[c]}$,

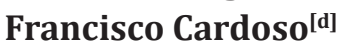

[a] $\mathrm{PhD}$, professora associada do Departamento de Fisioterapia da Universidade Federal de Minas Gerais (UFMG), Belo Horizonte, MG - Brasil, e-mail: fatimarp@globo.com

[b] MSc., Doutoranda do Programa de Pós-Graduação em Ciências da Reabilitação pela Universidade Federal de Minas Gerais (UFMG), Belo Horizonte, MG - Brasil, e-mail: lipia@bol.com.br

[c] $\mathrm{PhD}$, professora associada do Departamento de Fisioterapia da Universidade Federal de Minas Gerais (UFMG), Belo Horizonte, MG - Brasil, e-mail: lfts@eeffto.ufmg.br

[d] PhD, professor titular do Departamento de Clínica Médica da Universidade Federal de Minas Gerais (UFMG), Belo Horizonte, MG - Brasil, e-mail: cardosofe@terra.com.br

\section{Resumo}

Introdução: Indivíduos com doença de Parkinson (DP) apresentam déficits motores na marcha, postura e equilíbrio e tendem a apresentar redução mais acentuada do nível de atividade física do que indivíduos assintomáticos da mesma idade. 0 declínio físico pode estar associado à perda de força muscular, da capacidade física e piora do desempenho funcional na DP. Objetivo: Avaliar os efeitos de um programa de fortalecimento e condicionamento aeróbio no desempenho funcional e na capacidade física de indivíduos com DP. Materiais e métodos: Dezessete indivíduos, com média de idade de 60,35 \pm 9,94 anos e estágios I a III da Hoehn e Yahr, participaram de um programa de exercícios três vezes por semana durante 12 semanas. Avaliou-se a força muscular por meio do dinamômetro manual, o desempenho funcional por meio da Unified Parkinson Disease Rating Scale (UPDRS), avaliou-se também a velocidade da marcha e habilidade em usar escadas e a capacidade física por meio do perfil de atividade humana (PAH). Utilizou-se a estatística descritiva, testes t e ANOVA para medidas repetidas para a análise. Resultados e discussão: Os dorsoflexores bilaterais foram o único grupo muscular a apresentar maior torque após a intervenção ( $F=7,93 ; p=0,008)$. 
Houve ganho nas medidas de desempenho funcional: velocidade da marcha $(p=0,028)$, velocidade para subir $(p=0,001)$ e descer $(p=0,002)$ escadas, no escore total da UPDRS $(p=0,007)$ e do PAH $(p<0,0001)$. Considerações finais: $\mathrm{O}$ uso combinado de condicionamento aeróbio e fortalecimento muscular resultou em melhoras no desempenho funcional e na capacidade física de indivíduos com DP leve a moderada.

Palavras-chave: Doença de Parkinson. Fortalecimento muscular. Exercício aeróbio. Desempenho funcional.

\section{Abstract}

Introduction: Individuals with Parkinson's disease (PD) show gait, posture, and balance deficits and, compared to asymptomatic individuals of similar ages, demonstrate more accentuated decreases in physical activity levels. These physical declines could be associated with losses of muscular strength, physical capacity, and functional performance. Objectives: To investigate the effects of a combined program of muscular strengthening and aerobic conditioning on functional performance and physical capacity of individuals with PD. Materials and methods: Seventeen PD volunteers, who had a mean age of $60.35 \pm 9.94$ years on the stages 1 to 3 on the Hoehn and Yahr scale, participated in the program conducted three times/week during 12 weeks. The following outcome measures were obtained before and after the program: muscular strength with the hand-held dynamometer, functional performance with the Unified Parkinson Disease Rating Scale (UPDRS), gait speed, ability to manage stairs, and physical capacity with the human activity profile (HAP). Descriptive statistics, $t$-tests, and repeated measure ANOVAS were employed for analyses. Results and discussion: The bilateral dorsal flexors were the only group which demonstrated significant increases in torque production after the intervention ( $F=7.93 ; p=0.008)$. However, significant gains were observed for all measures of functional performance: Gait speed ( $p=0.028)$, stair ascent ( $p=0.001)$, stair descent ( $p=0.002)$, total UPDRS score $(p=0.007)$, and HAP score $(p<0.0001)$. Conclusions: The combined program of muscular strengthening and aerobic conditioning resulted in improvements in measures of functional performance and physical activity levels in individuals with $P D$ in the early and moderate stages of the disease.

Keywords: Parkinson's disease. Muscular strengthening. Aerobic exercise. Functional performance.

\section{Introdução}

Com a progressão da enfermidade, indivíduos com a doença de Parkinson (DP) apresentam déficits motores na marcha, na postura e no equilíbrio (1). Tais déficits contribuem para a perda de independência, quedas e inatividade, com consequente isolamento social e maior risco de osteoporose e doenças cardiovasculares $(2,3)$. Ainda que a abordagem medicamentosa seja o tratamento padrão para a DP, a medicação apresenta perda da eficácia com o tempo e está associada ao desenvolvimento de complicações motoras típicas, como discinesias (4). Tem sido sugerido que os exercícios físicos podem estimular o controle do movimento e retardar a progressão da doença $(5,6)$.

A inatividade é considerada um fator importante para a deterioração dos sintomas e patogênese na DP (7). Estudos têm demonstrado que indivíduos com incapacidades são menos ativos fisicamente que in- divíduos sem incapacidades (7). 0 estudo de Goulart et al. (8) observou que indivíduos em estágios inicial e moderado da DP tendem a apresentar redução mais acentuada do nível de atividade física que indivíduos assintomáticos da mesma idade. Embora as causas da inatividade ainda não estejam esclarecidas, o declínio físico tem sido associado à perda de força muscular, da capacidade física e piora do desempenho funcional em indivíduos com DP $(3,9,10)$. Em tais indivíduos, a redução da força muscular pode comprometer o equilíbrio e a habilidade para realizar atividades de vida diária como caminhar ou levantar-se de uma cadeira $(11,12)$. Assim como na função muscular, indivíduos com DP apresentam perdas na função cardiorrespiratória $(3,13,14)$. Tais sujeitos apresentam níveis similares de capacidade aeróbia máxima quando comparados a indivíduos assintomáticos, porém, o pico máximo ocorre em intensidades menores de exercício sugerindo baixa eficiência metabólica (13). Estudos recentes utilizando modelos de animais com 
DP demonstraram que exercícios aeróbios podem produzir efeito neuroprotetor e promover restauração de vias neuronais comprometidas na DP $(15,16)$.

A literatura tem demonstrado que programas de atividade física envolvendo fortalecimento muscular e condicionamento aeróbio resultam em melhoras no desempenho motor de indivíduos com sequelas neurológicas, como em hemiplégicos $(17,18)$. Alguns estudos examinaram os efeitos isolados dessas intervenções na DP (19-21), entretanto, o uso combinado de tais intervenções não foi investigado na DP. Considerando os déficits nos sistemas musculoesquelético e cardiorrespiratório de indivíduos com DP, o objetivo do presente estudo foi avaliar os efeitos de 12 semanas de um programa combinado de exercícios aeróbios e de fortalecimento muscular no desempenho motor e na capacidade física e funcional de indivíduos leve a moderadamente afetados pela DP.

\section{Materiais e métodos}

Este foi um estudo com desenho quase-experimental, sendo os indivíduos avaliados antes e após o programa de treinamento.

\section{Participantes}

Os indivíduos foram recrutados no Ambulatório de Distúrbios do Movimento da Universidade e incluídos no estudo de acordo com os seguintes critérios: ser portador de DP idiopática diagnosticada pelo neurologista; estar nos estágios 1 a 3 na escala de Hoehn e Yahr modificada (22); ter idade acima de 40 anos; não apresentar outra doença neurológica e nem alterações ortopédicas ou cardiológicas limitantes; não apresentar problemas cognitivos graves que impedissem a compreensão dos testes e dos exercícios; não estar praticando atividade física regular, apresentar um atestado cardiológico de aptidão física e assinar o Termo de Consentimento Livre e Esclarecido aprovado pelo Comitê de Ética em pesquisa da instituição (Parecer 045/99).

Instrumentos de avaliação

Para caracterização da amostra, foram coletados dados relativos à idade, sexo, tempo de evolução da doença e estágios de incapacidade Hoehn \& Yahr modificada. Todos os indivíduos com DP estavam em uso regular de medicação antiparkinsoniana e foram avaliados sistematicamente no período "ON", cerca de uma hora após a ingestão medicamentosa.

Para avaliação da força muscular, utilizou-se o dinamômetro manual (Nicholas Manual MuscleTesterModel 01160). Foi avaliada a força isométrica máxima, sustentada por cinco segundos, dos seguintes grupos musculares: flexores dorsais e plantares e flexores e extensores do joelho e quadril. Esse instrumento fornece medidas em quilogramas-força (kgf), que foram convertidas em Newton-metro $(\mathrm{N} \cdot \mathrm{m})$ com correção da gravidade. Para a conversão do torque produzido contra o torque gerado pela gravidade, a seguinte fórmula foi utilizada: força $(\mathrm{kgf}) \times 9,81 \times$ distância do eixo articular (metros) + massa do segmento $(\mathrm{kg}) \times 9,81 \times$ distância do centro de massa do segmento (m); e, quando a ação foi realizada sem influência da gravidade, a fórmula utilizada para a conversão foi: força $(\mathrm{kgf}) \times$ 9,81 × distância do eixo articular $(\mathrm{m})$. As medidas antropométricas utilizadas foram as propostas por Winter (23). Bohannon (24) encontrou valores de confiabilidade do dinamômetro manual de 0,97 a 0,98 com índice de significância de $\mathrm{p}<0,01$, mostrando que esse é um instrumento fidedigno para avaliar o torque muscular. Foi realizada uma comparação das medidas de torque entre um lado e outro do corpo, sendo o lado mais afetado definido como aquele com o maior número de grupos musculares apresentando menor torque.

Para a avaliação da velocidade da marcha, solicitou-se aos indivíduos que deambulassem em uma velocidade autosselecionada, em um corredor de 16 metros. Foi cronometrado o tempo gasto para percorrer os doze metros centrais, visto que os dois metros iniciais e os dois finais foram considerados como fase de aceleração e desaceleração, respectivamente, e, portanto, foram descartados. Três medidas foram coletadas por meio de um cronômetro digital e a média entre elas foi utilizada para análise. Medidas de velocidade apresentam um alto índice de confiabilidade tanto em ambientes domésticos como em ambientes clínicos e laboratoriais $(25,26)$.

A habilidade para utilizar escadas é uma medida importante da mobilidade funcional e o tempo utilizado para subir um determinado lance de escadas tem sido reconhecido como uma medida eficaz (27). Foi cronometrado o tempo gasto pelo indivíduo para subir e para descer, em uma velocidade 
confortável, um lance de escada com cinco degraus de aproximadamente $15 \mathrm{~cm}$ cada, sendo permitido o uso do corrimão, se necessário. Três medidas foram coletadas e a média entre elas foi calculada. O tempo foi obtido em segundos e, posteriormente, transformado em minutos para que a cadência fosse obtida em degraus/minuto, seguindo o protocolo, descrito por Olney et al. (27), que apresenta um índice de confiabilidade entre examinadores de 0,90 com indivíduos saudáveis.

A avaliação clínico-funcional dos indivíduos foi realizada por meio da Unified Parkinson's Disease Rating Scale (UPDRS) que é composta por quatro dimensões: atividade mental, comportamento e humor; atividades de vida diária; atividade motora e complicações medicamentosas. Cada item é subdividido em cinco subitens, que variam de zero a quatro, e quanto maior o escore pior o estado do paciente. 0 item referente a complicações medicamentosas não foi utilizado neste estudo (28).

0 perfil de atividade humana (PAH) é utilizado na avaliação do nível funcional e de atividade física de indivíduos em qualquer faixa etária com ou sem disfunção (29). 0 instrumento é composto por 94 itens de atividades de diferentes níveis de dificuldades de acordo com o custo energético. Baseando-se em cada resposta, calcula-se o escore máximo de atividade e o escore ajustado de atividade (EAA), que representa os níveis médios de equivalentes metabólicos gastos em um dia típico. O PAH teve sua adaptação transcultural para a população brasileira realizada por Souza et al. (30).

\section{Protocolo de treinamento}

Os indivíduos participaram de um programa de atividade física realizado três vezes por semana durante 12 semanas. Cada sessão teve duração de 75 minutos, sendo subdividida em: 15 minutos de alongamento e mobilidade de tronco e membros; 20 minutos de exercícios de fortalecimento muscular de tronco e membros inferiores (MMII) utilizando caneleiras e bastões; 30 minutos de exercícios aeróbios, utilizando stepping e caminhada, com a manutenção da frequência cardíaca entre $65 \%$ e $80 \%$ da frequência cardíaca máxima de acordo com a idade (31); e 10 minutos de alongamento e relaxamento muscular. A frequência cardíaca foi constantemente monitorada por meio de cardiofrequencímetro (Polar
T31) e a pressão arterial foi medida ao início e ao término de cada sessão de treinamento.

0 programa de fortalecimento muscular combinou exercícios isométricos e isotônicos para os principais grupos musculares do tronco (abdominais e paravertebrais) e dos MMII (flexores e extensores do quadril e joelho, dorsiflexores e flexores plantares do tornozelo). Foram realizadas duas séries de 10 repetições para cada grupo muscular com intervalo de repouso entre elas. A carga utilizada respeitou o potencial inicial de cada paciente, sendo reajustada com acréscimos de $0,5 \mathrm{~kg}$ de acordo com sua evolução. Os pacientes foram orientados a realizar o movimento contra a resistência determinada, manter a contração por seis segundos e retornar.

\section{Análise estatística}

Uma estatística descritiva foi realizada para as variáveis propostas por meio do programa SPSS para Windows (versão 13.0). Utilizou-se a análise de variância (ANOVA) para medidas repetidas com dois níveis fatoriais, sendo um deles o fator tempo (antes e após a intervenção); e o outro, membro inferior (mais e menos afetado), foi utilizado para avaliar o torque dos músculos flexores, extensores do quadril, joelho e tornozelo. Análises de contrastes foram usadas para localizar os pares específicos entre os quais as diferenças foram significativas. 0 nível de significância foi estabelecido em $\alpha \leq 0,05$. Testes t-pareados foram utilizados para avaliar o efeito do treinamento nas medidas de desempenho funcional e capacidade física.

\section{Resultados}

Participaram do presente estudo 17 indivíduos com DP, sendo 5 mulheres e 12 homens, com idade entre 41 e 77 anos e tempo médio de evolução da doença variando entre 1 e 13 anos (Tabela 1).

A ANOVA demonstrou não haver diferença estatisticamente significativa entre os períodos de tempo (pré e pós-intervenção) e entre lado mais e menos afetado para a variável torque dos flexores e extensores de quadril e joelho e flexores plantares do tornozelo. 0 torque dos flexores dorsais de ambos os lados foi significativamente maior após a intervenção ( $F=7,93 ; p=0,008)$ (Tabela 2). 
Tabela 1 - Caracterização dos indivíduos com doença de Parkinson participantes do estudo

\begin{tabular}{lccccl}
\hline Indivíduos & Idade (anos) & Sexo & Tempo de evolução (anos) & Hoehn e Yahr & Medicação em uso \\
\hline 1 & 44 & M & 2 & 1,0 & Sinemet, Biperideno \\
2 & 72 & M & 12 & 2,0 & Tasmax, Cronomet \\
3 & 55 & F & 5 & 3,0 & Niar, Artane, Mantidan \\
4 & 58 & M & 3 & 2,0 & Sinemet, Mantidan \\
5 & 69 & M & 5 & 2,5 & Akineton, Mantidan, Bagren \\
6 & 56 & F & 6 & 3,0 & Mantidan, Akineton \\
7 & 66 & F & 5 & 1,0 & Sinemet, Bromocriptina \\
8 & 54 & M & 3 & 1,0 & Akineton, Niar, Mantidan \\
9 & 63 & F & 4 & 2,5 & Sinemet \\
10 & 41 & M & 1 & 1,0 & Mantidan, Biperideno \\
11 & 51 & M & 8 & 2,5 & Artane, Bromocriptina, Sinemet \\
12 & 60 & M & 13 & 1,5 & Sinemet, Mantidan, Bagren \\
13 & 69 & M & 9 & 1,5 & Sinemet, Mantidan \\
14 & 77 & M & 3 & 2,0 & Sinemet \\
15 & 69 & M & 4 & 2,0 & Sinemet \\
16 & 57 & M & 6 & 2,5 & Sinemet, Biperidina, Amitriptilina, \\
17 & 66 & F & 6 & 3,0 & Sinemet, Akineton \\
\hline Média (DP) & $60,4(9,8)$ & - & $5,5(3,2)$ & - & - \\
\hline
\end{tabular}

Legenda: $\mathrm{M}=$ masculino; $\mathrm{F}=$ feminino; $\mathrm{DP}=$ desvio-padrão.

Fonte: Dados da pesquisa.

Tabela 2 - Estatística descritiva (média e desvio-padrão) das medidas de força muscular (em N · m) avaliadas antes e após 0 programa de treinamento

\begin{tabular}{lccccc}
\hline & \multicolumn{2}{c}{ MI mais afetado } & & \multicolumn{2}{c}{ MI menos afetado } \\
\cline { 2 - 3 } \cline { 5 - 6 } Grupo muscular & Antes & Após & & Antes & Após \\
\hline Flexão dorsal & $23,48 \pm 13,25$ & $34,50 \pm 16,14^{*}$ & & $30,81 \pm 20,46$ & $34,51 \pm 15,98^{*}$ \\
Flexão plantar & $35,72 \pm 21,08$ & $43,67 \pm 18,34$ & & $43,24 \pm 23,44$ & $44,67 \pm 15,35$ \\
Extensão de joelho & $60,33 \pm 36,03$ & $62,95 \pm 21,31$ & & $70,73 \pm 35,56$ & $67,93 \pm 21,33$ \\
Flexão de joelho & $47,44 \pm 26,90$ & $46,92 \pm 24,09$ & & $54,88 \pm 28,19$ & $53,28 \pm 28,22$ \\
Extensão de quadril & $88,30 \pm 52,53$ & $117,30 \pm 43,82$ & & $106,30 \pm 59,47$ & $127,43 \pm 54,10$ \\
Flexão de quadril & $136,11 \pm 53,90$ & $140,07 \pm 42,11$ & & $154,28 \pm 54,15$ & $149,69 \pm 40,82$ \\
Força isométrica total & $528,71 \pm 222,7$ & $608,22 \pm 189,32$ & & $621,91 \pm 247,92$ & $633,40 \pm 196,55$ \\
\hline
\end{tabular}

Legenda: $\mathrm{Ml}=$ membro inferior; ${ }^{*}=\mathrm{p}<0,05$.

Fonte: Dados da pesquisa.

A velocidade da marcha aumentou significativamente, de $1,11 \pm 0,23 \mathrm{~m} / \mathrm{s}$ para $1,22 \pm 0,17 \mathrm{~m} / \mathrm{s}$, no período pós intervenção $(\mathrm{p}=0,028)$. Houve ganho significativo na habilidade para subir $(p=0,001)$ e para descer $(p=0,002)$ escadas, no escore da UPDRS total $(p=0,007)$ e no nível de atividade física realizada pelos indivíduos ( $p<0,0001)$ após o programa de treinamento (Gráficos 1 e 2). 


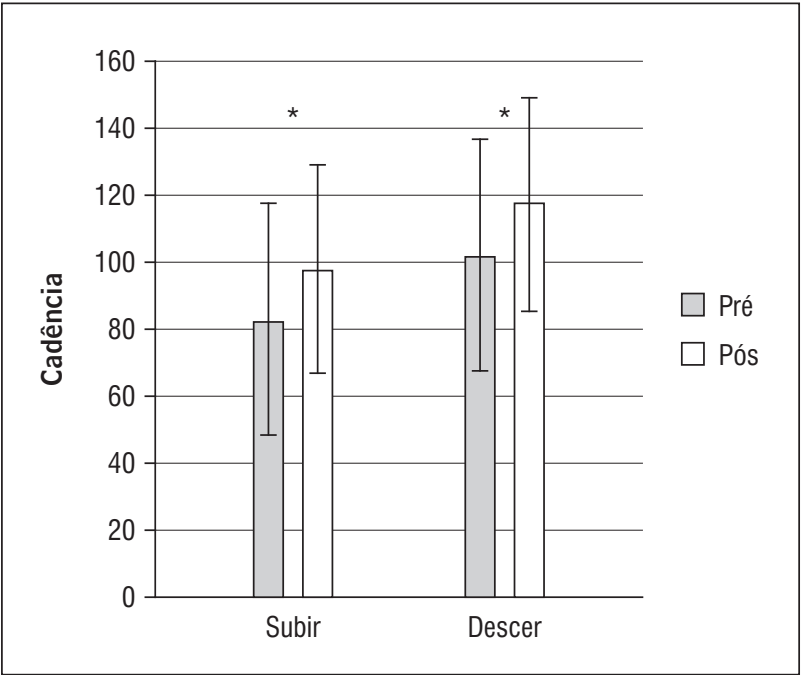

Gráfico 1 - Média e desvio-padrão da velocidade para usar escadas antes e após o programa de treinamento

Legenda: * $=p<0,05$.

Fonte: Dados da pesquisa.

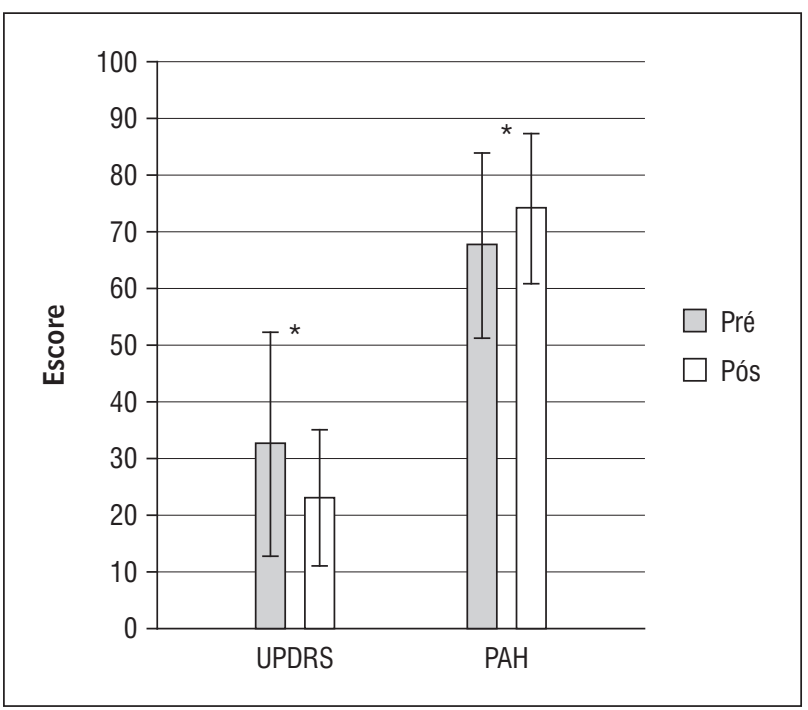

Gráfico 2 - Média e desvio-padrão do escore total da UPDRS e do escore ajustado de atividade do PAH antes e após o programa de treinamento

Legenda: ${ }^{*}=p<0,05$; UPDRS $=$ Unified Parkinson's Disease

Rating Scale. $\mathrm{PAH}=$ Perfil de Atividade Humana.

Fonte: Dados da pesquisa.

\section{Discussão}

No presente estudo, os indivíduos com DP apresentaram ganhos físicos e funcionais após serem submetidos a um programa de exercícios associando fortalecimento muscular e condicionamento aeróbio.
Em relação à função muscular, não houve diferença no torque da maioria dos grupos musculares dos membros mais e menos afetados após o programa de treinamento. Apenas o torque dos flexores dorsais foi maior em ambos os lados após a intervenção. É possível que esse achado se relacione à intensidade da carga utilizada. No presente estudo, a carga não foi estabelecida a partir de um teste de resistência máxima, uma vez que vários indivíduos só podiam realizar os movimentos, inicialmente, contra a força da gravidade. Esse aspecto pode ter contribuído para que a sobrecarga, bem como sua progressão, tenha sido subestimada, impedindo assim os estímulos necessários para as modificações neurais e musculares advindas do treinamento. Similarmente, um programa de fortalecimento muscular por meio de thera-bands não demonstrou ganhos nas medidas de força muscular do joelho de indivíduos com DP (32). Para os autores, a alta variabilidade das medidas e a baixa intensidade de exercícios têm sido associadas à ausência de ganhos de força muscular em indivíduos com DP (32).

É interessante notar que, mesmo que a intensidade utilizada não tenha produzido modificações musculares na maioria dos grupos musculares trabalhados, os flexores dorsais apresentaram ganhos significativos no período pós-intervenção. Alguns aspectos podem ter contribuído para esse resultado. Primeiro, o uso das caneleiras para o fortalecimento muscular foi mantido nos MMII no momento da caminhada com a finalidade de manter a frequência cardíaca adequada para o treinamento aeróbio. Esse aspecto pode ter aumentado o tempo de sobrecarga dos flexores dorsais, levando aos ganhos observados. Segundo, ainda que todos os indivíduos com DP do presente estudo não possam ser considerados idosos, a literatura reporta um declínio progressivo da força muscular no tornozelo em relação às demais articulações, especificamente nos flexores dorsais, o que pode ter contribuído para que essa musculatura seja mais suscetível a uma sobrecarga localizada como a que foi utilizada (3335). Considerando que a força muscular dos flexores dorsais é um componente essencial para a realização de diferentes atividades funcionais $(36,37)$, o aumento do torque muscular observado pode ter contribuído, ainda que parcialmente, para a melhora funcional observada.

A literatura tem demonstrado ganhos funcionais na DP por meio do treinamento aeróbio. Um estudo 
recente comparou o desempenho na marcha entre dois grupos de indivíduos com DP submetidos a um protocolo de treino aeróbio e de exercícios sensoriais (19). Os resultados demonstraram que apenas o grupo submetido ao condicionamento aeróbio obteve melhora significativa na velocidade da marcha e no comprimento do passo. Acredita-se que tais resultados se devam ao fortalecimento muscular dos membros inferiores advindo do treinamento aeróbico (19). É possível que a utilização de medidas de avaliação de torque isométrico não tenha sido sensível e específica para verificar a contribuição do treino aeróbio no desempenho muscular dos indivíduos do presente estudo. Ainda assim, independente do mecanismo, o treinamento aeróbio contribuiu para a melhora física e funcional encontrada nos indivíduos com DP deste estudo. Assim, no contexto de uma doença degenerativa e progressiva como a DP, os benefícios clinicamente importantes justificam o protocolo utilizado.

Embora a literatura aponte que o condicionamento físico é reduzido em indivíduos com DP (3, 13,14), há uma escassez de estudos que abordem o treinamento aeróbio, bem como medidas que possam ser utilizadas para avaliar a capacidade física na DP. Uma forma simples de se avaliar o nível funcional e de atividade física, tanto para indivíduos saudáveis quanto para aqueles com algum grau de disfunção, é por meio do PAH. Trata-se de um instrumento simples e de fácil aplicação que fornece uma medida rápida e significativa da alteração dos níveis de energia e possibilita comparações entre os níveis de atividade de distintas populações $(29,30)$.

Os resultados do presente estudo demonstraram melhora significativa no EAA, que é um dos escores primários do PAH. Esse achado indica que, após a intervenção, os indivíduos do presente estudo passaram a desempenhar atividades que não realizavam mais, ou seja, houve uma diminuição no número de itens da PAH que o indivíduo identificava como incapaz de fazer. $\mathrm{O}$ aumento significativo no EAA indica melhora do nível de energia desses indivíduos em razão da capacidade de executar atividades que demandam maior gasto energético, provavelmente como consequência de uma melhora da capacidade física e funcional após o programa de treinamento.

Um estudo recente de Schenkman et al. (14) apontou ganhos significativos no condicionamento físico e no escore da UPDRS após 16 semanas de treinamento aeróbico na esteira com controle sistemático da frequência cardíaca. Para os autores, esse tipo de intervenção permite outros benefícios motores, como observado por meio da UPDRS. De acordo com Schrag et al. (38), diferenças mínimas de cinco pontos no domínio "atividade motora" e de dois pontos no domínio "atividade de vida diária" da UPDRS são clinicamente relevantes. No presente estudo, as diferenças observadas entre as médias dos domínios foram de seis pontos no domínio "atividade motora" e de quatro pontos no domínio "atividade de vida diária" da UPDRS, sugerindo que o programa combinado de condicionamento aeróbio e fortalecimento muscular em grupo foi capaz de provocar mudanças clínicas e funcionais importantes, como medido por tal escala específica para a DP.

Embora os resultados sejam importantes, o presente estudo apresenta limitações. Trata-se de um desenho metodológico do tipo quase-experimental, o que impede uma relação de causa e efeito em um grupo de variáveis (39). Além disso, a generalização dos resultados para a população de indivíduos com DP é dificultada em razão de dois fatores principais. Em primeiro lugar, a amostra de conveniência do estudo foi constituída por indivíduos motivados e assíduos do setor de tratamento, caracterizando, assim, um grupo altamente engajado com a intervenção. É possível que a baixa condição socioeconômica desses sujeitos justifique a alta assiduidade demonstrada, pois a participação nesse tipo de pesquisa pode ser a única oportunidade de acesso a tratamentos gratuitos, para indivíduos de baixa renda, no contexto da realidade brasileira. Em segundo lugar, tratase da própria natureza da intervenção. Os achados do estudo não podem ser generalizados para indivíduos mais gravemente afetados pela DP (HY acima de 3). A intervenção proposta requer indivíduos com maior mobilidade e certa independência, como aqueles leve a moderadamente afetados (HY $=1 \mathrm{a}$ 3 ) incluídos neste estudo. Outro fator a ser mencionado é que foram avaliados aqui apenas os efeitos imediatos da intervenção combinada. Considerando a característica progressiva da doença, são necessários estudos aleatorizados e controlados para, definitivamente, estabelecer a eficácia, assim como os efeitos em longo prazo, desse tipo de intervenção.

Contudo, os resultados do presente estudo são relevantes para a prática do fisioterapeuta que atua com indivíduos com DP. Ainda que não tenham sido observadas modificações nas medidas de torque muscular, depois de 12 semanas foram observadas 
melhoras funcionais, clínicas e no nível de atividade física dos pacientes, como avaliado por meio da marcha, habilidade em usar escadas e das escalas UPDRS e PAH. Além disso, a intervenção utilizada aqui também proporcionou ganhos significativos de qualidade de vida, medidos por meio do perfil de saúde de Nottingham (PSN) (40). Além do ganho obtido no escore total do PSN, os principais ganhos observados foram nos domínios de reação emocional, interação social e habilidade física (40). Portanto, é possível que o programa de treinamento, associando condicionamento aeróbio e fortalecimento muscular em grupo aumente os níveis de energia, bem como proporcione oportunidades de socialização e mudanças nas alterações emocionais comuns em indivíduos com DP.

Além dos benefícios já citados, uma importante vantagem a ser considerada é a fácil aplicação do programa de exercícios proposto no uso clínico do fisioterapeuta para o tratamento da DP. 0 protocolo combina técnicas simples e não requer equipamentos caros e complexos, podendo ser facilmente inserido como abordagem terapêutica em clínicas, consultórios ou mesmo no domicílio. Vale destacar também a importância da progressão sistemática da frequência cardíaca de treinamento, visto que esse controle garante que as adaptações necessárias no sistema cardiorrespiratório e periférico sejam obtidas gradativamente. Sendo assim, o protocolo utilizado e os resultados obtidos neste estudo podem auxiliar e guiar os profissionais que trabalham com indivíduos com DP e que utilizam exercícios físicos como meio de intervenção.

\section{Conclusões}

O uso combinado de condicionamento aeróbio e fortalecimento muscular resultou em melhoras nas medidas de desempenho funcional e de capacidade física de indivíduos em fase leve a moderada de evolução da DP. 0 protocolo de exercícios utilizado é de fácil aplicação e demonstrou ganhos na velocidade da marcha, na habilidade em usar escadas, no nível de atividade física e nos sintomas clínicos apresentados por tais indivíduos. A melhora da mobilidade, da capacidade física e o aumento da socialização entre indivíduos com DP justificam o uso de programas específicos de atividade física como estratégia de reabilitação nessa população.

\section{Agradecimentos}

Agradecemos à FAPEMIG/CNPq pelo apoio recebido.

\section{Referências}

1. Keus SHJ, Bloem BR, Hendriks EJM, Bredero-Cohen AB, Munneke M. Evidence-based analysis of physical therapy in Parkinson's disease with recommendations for practice and research. Mov Disord. 2007; 22(4):451-60.

2. Garret NA, Brasure M, Schimtz KH, Schultz MM, Huber MR. Physical inactivity: direct cost to a health plan. Am J Prev Med. 2004;27(4):304-9.

3. Canning CG, Alison JA, Allen NE, Groller H. Parkinson's disease: an investigation of exercise capacity, respiration function, and gait. Arch Phys Med Rehabil. 1997; 78(2):199-207.

4. Fabbrini G, Brotchie JM, Grandas F, Nomoto M, Goetz CG. Levodopa-induced dyskinesias. Mov Disord. 2007; 22(10):1379-89.

5. Robichaud JA, Corcos DM. Motor deficits, exercise and Parkinson's disease. Quest. 2005;57(1):85-107.

6. Crizzle AM, Newhouse IJ. Is physical exercise beneficial for persons with Parkinson's disease? Clin J Sport Med. 2006;16(5):422-5.

7. Hirsch MA, Farley BG. Exercise and neuroplasticity in persons living with Parkinson's disease. Eur J Phys Rehabil Med. 2009;45(2):215-29.

8. Goulart F, Santos CC, Teixeira-Salmela LF, Cardoso F. Análise do desempenho funcional em pacientes portadores de doença de Parkinson. Acta Fisiátrica. 2004;11(1):12-6.

9. Morris ME. Movement disorders in people with Parkinson disease: a model for physical therapy. Phys Ther. 2000;80(6):578-97.

10. Glendinning DS. A rationale for strength training in patients with Parkinson's disease. Neurol Rep. 1997; 21:132-35.

11. Inkster LM, Eng JJ, Macintyre DL, Stoessl AJ. Leg muscle strength is reduced in Parkinson's disease and relates to the ability to rise from a chair. Mov Disord. 2003;18(2):157-62. 
12. Pääsuke M, Ereline J, Gapeyeva H, Joost K, Mõttus K, Taba P. Leg-extension strength and chair-rise performance in elderly women with Parkinson's disease. J Aging Phys Act. 2004;12(4):511-24.

13. Protas EJ, Stanley RK, Jankovic J, Macneill B. Cardiovascular and metabolic responses to upper and lower extremity exercise in men with idiopathic Parkinson's disease. Phys Ther. 1996;76(1):34-40.

14. Schenkman M, Hall D, Kumar R, Kohrt WM. Endurance exercise training to improve economy of movement of people with Parkinson disease: three case reports. Phys Ther. 2008;63(1):63-76.

15. Pothakos K, Kurz M, Lau YS. Restorative effect of endurance exercise on behavioral deficits in the chronic mouse model of Parkinson's disease with severe neurodegeneration. BMC Neurosci. 2009;10(6):1-46.

16. Fisher BE, Petzinger GM, Nixon K, Hogg E, Bremmer $\mathrm{S}$, Meshul CK, et al. Exercise-induced behavioral recovery and neuroplasticity in the 1-methyl-4-pheny1,2,3,6-tetrahydropyridine-lesioned mouse basal ganglia. J Nerousci Res. 2004;77(3):378-90.

17. Teixeira-Salmela LF, Olney SJ, Nadeau S, Brower B. Reducing impairment and disability in chronic stroke survivors through muscle strengthening and physical conditioning. Arch Phys Med Rehabil. 1999; 80(10):1211-18.

18. Teixeira-Salmela LF, Nadeau S, McBride I, Olney SJ. Effects of muscle strengthening and physical conditioning training on temporal, kinematic and kinetic variables during gait in chronics stroke survivors. J Rehabil Med. 2001;33(2):53-60.

19. Sage MD, Almeida QJ. Symptom and gait changes after sensory attention focused exercise vs aerobic training in Parkinson's disease. Mov Disord. 2009;24(8): 1132-38.

20. Scandalis TA, Bosak A, Berliner JC, Helman LL, Wells MR. Resistance training and gait function in patients with Parkinson's disease. Am J Phys Med Rehabil. 2001;80(1):38-43.

21. Dibble LE, Hale TF, Marcus RL, Droge J, Gerber JP, Lastayo PC. High-intensity resistance training amplifies muscle hypertrophy and functional gains in persons with Parkinson's disease. Mov Disord. 2006;21(9):1444-52.

22. Hoehn MM, Yahr MD. Parkinsonism: onset, progression and mortality. Neurology. 1967;17(5):427-42.
23. Winter DA. Anthropometry. In: Winter DA. Biomechanics and motor control of human movement. 2nd ed. New York: Wiley Interscience Publication; 1990. p. 51-74.

24. Bohannon RW. Test-retest reability of hand-held dynamometry during a single session of strength as sessment. Phys Ther. 1996;66(2):206-9.

25. Collen F, Wade D, Bradshaw C. Mobility after stroke: reliability of measures of impairment and disability. Int Disabil Stud. 1990;12(1):6-9.

26. Richards C, Olney S. Hemiplegic gait following stroke. Part II: recovery and physical therapy. Gait Posture. 1996;4(2):149-62.

27. Olney S, Elkin N, Lowe P. An ambulation profile for clinical gait evaluation. Physiol Can. 1979;31:85-90.

28. Martínez-Martín P, Gil-Nagel A, Gracia LM, Gómez JB, Martínez-Sarriés J, Bermejo F. Unified Parkinson's disease rating scale characteristics and structure. Mov Disord. 1994;9(1):76-83.

29. Packer TL, Sauriol A, Brouwer B. Fatigue secondary to chronic illness: post polio syndrome, chronic fatigue syndrome, and multiple sclerosis. Arch Phys Med Rehabil. 1994;75(10):1122-6.

30. Souza AC, Magalhães LC, Teixeira-Salmela LF. Adaptação transcultural e análise das propriedades psicométricas da versão brasileira do perfil de atividade humana. Cad Saúde Pública. 2006;22(12):2623-36.

31. Pollock M, Graves J, Swart D, Rosenthal DT. Exercise training and prescription for the elderly. South Med J. 1994;87(5):S88-95.

32. Pedersen SW, Insulaner OA, Vretman M. Group training in Parkinsonism: quantitative measurements of treatment. Scand J Rehabil Med. 1990;22(4):207-11.

33. Whipple RH, Woltson LL, Amerman PM. The relationship of knee and ankle weakness to falls in nursing home residents: an isokinetic study. J Am Geriatr Soc. 1987;35(1):13-20.

34. Studenski S, Duncan PW, Chandler J. Postural responses and effector factors in persons with unexplained falls: results and methodology issues. J Am Geriatr Soc. 1991;39(3):229-34.

35. Wolfson L, Judge J, Whipple R, King M. Strength is a major factor in balance, gait, and the occurrence of falls. J Gerontol A Biol Sci Med Sci. 1995;50(Spec No):64-7. 
36. Lord SR, Murray SM, Chapman K, Munro B, Tiedemann A. Sit-to-stand performance depends on sensation, speed, balance and psychological status in addition to strength in older people. J Gerontol A Biol Sci Med Sci. 2002;57(8):M539-43.

37. Menz HB, Morris ME, Lord SR. Foot and ankle characteristics associated with impaired balance and functional ability in older people. J Gerontol A Biol Sci Med Sci. 2005;60:1546-52.

38. Schrag A, Sampaio C, Counsell N, Poewe W. Minimal clinically important change on the unified Parkinson's disease rating scale. Mov Disord. 2006;21(8):1200-7.

39. Portney LG, Walkins MP. Foundations of clinical research: applications to practice. 2nd ed. New Jersey: Prentice Hall Health; 2000.
40. Rodrigues-de-Paula F, Teixeira-Salmela LF, Farias CD, Brito PR, Cardoso F. Impact of an exercise program on physical, emotional, and social aspects of quality of life of individuals with Parkinson's disease. Mov Disord. 2006;21(8):1073-7.

Recebido: 03/01/2011

Received: 01/03/2011

Aprovado: 24/03/2011

Approved: 03/24/2011 